\title{
Progress in digitization of astronomical plate archives
}

\author{
René Hudec ${ }^{1,2,3 * \dagger}$ \\ ${ }^{1}$ Czech Technical University in Prague, Faculty of Electrical Engineering, \\ Technicka 2, CZ 16000 Prague, Czech Republic \\ ${ }^{2}$ Astronomical Institute of the Academy of Sciences of the Czech Republic \\ Fricova 298 - CZ 25165 Ondrejov, Czech Republic \\ ${ }^{3}$ Kazan Federal University, Kazan, Russian Federation \\ E-mail: rene.hudec@gmail.com
}

We report here on the progress in digitization of astronomical plate archives and tests of the scanning devices for digitization of archival astronomical plates. These archives represent large database of stellar, solar, and planetary astronomical data, astrometric, photometric, and spectroscopic, may be useful for numerous applications in modern astrophysics.

Frontier Research in Astrophysics - III (FRAPWS2018)

28 May - 2 June 2018

Mondello (Palermo), Italy

* Speaker.

$\dagger$ 


\section{Introduction}

Before the era of electronic image recording devices, the photography was the only method available to record images and spectra in astronomy. As a result, very extended and numerous collections of astronomical photographic records exist at many worlds observatories and astronomical institutes (Hudec 1999 and 2005). My estimate based on personal visits and works with many different plate collections (more than 50) is that there are more than 10 million astronomical photographic negatives (on glass and plastic substrates) worldwide (Hudec, 1999, Hudec 2005, Hudec and Hudec, 2013).

However, the access to the data recorded in photographic archives is, in principle, difficult and laborious. Digitization is a necessary step for an extended evaluation of the plate data using dedicated programs and computers.

The astronomical plate archives represent the only method how to study the behavior of celestial objects over very long (100 years or even more) time intervals (Hudec, 1999 and Hudec et al., 2012). In addition, huge monitoring times allows to detect and to study rare events such as flares and flashes. The spectral databases allow to study prominent spectra and/or spectral changes as well (Hudec et al., 2012).

There are various image/target types recorded on the photographic negatives. The basic separation is between direct images and spectral images, where the spectral images can be either single or multiple spectra, or wide field low dispersive spectra with objective prism. The direct images may be single (each star one image) or multiple (each star is recorded several times on the same plate, a technique used in the past e.g. for searches for rapid variability). The negatives were in the past used with large number and rich variety of astronomical telescopes and cameras, and correspondingly, the type variety of archived negatives is also very rich. Many large collections represent wide field sky images, but there are also extended collections of small sized negatives, both glass and plastics, taken in the focal plates of medium and large telescopes. While the large part of astronomical negatives worldwide covers sky regions with stellar fields, there are also many extended collections with Sun, Moon, and planetary images.

It should be noted that the photography served as major recording medium not only in astronomy but also in many other areas of science and technology and in addition to that served as medium to record images in national archives, various museum etc. Before era of Xerox copy machines, the photography was the widely used method to make copies of documents, and these negatives are stored in large museum and cultural collections as well. Many problems related to astronomical plate archives such as suitable storage, digitization, defects, degradation, etc., are common for all photographic archives.

\section{The astronomical photographic plate archives}

The astronomical archival plate collections that we visited and investigated during last years include those housed in the following 16 US institutions: Carnegie Observatories Pasadena, CA, Lick Observatory, CA Yerkes Observatory, WI, Mt Palomar Observatory, CA, PARI, Rosman, NC (which has a collection of plates from many observatories), KPNO Tucson, AZ, CFHT Waimea, HI, IfA Manoa, HI, USNO Flagstaff, AZ, USNO Washington, DC, Steward Observatory Tucson, 
AZ, NMSU, Las Cruces, NM, Rosemary Hill Observatory, University of Florida, Gainesville, FL And Leander McCormick Observatory, University of Virginia, Charlottesville, VA, Smithsonial Archives Washington, DC, and Hazy Space Center, Dulles, VA. Our estimate is that there are more than 2 million astronomical archival plates and/or negatives in these archives. There are however many additional US plate collections not included in our study (Robbins and Osborn, 2009). During our personal visits, we performed a quality check and analyzed these plate archives with emphasis on their scientific, historical and cultural value, which we have found to be enormous. In addition to that, we have visited and digitized samples of astronomical photographic databases in Europe, e.g. in Torino, Vatican Castel Gandolfo, Catania, Merate, Asiago, Bologna, Loiano, OHP Haute Provence, Nice/Calern, Tuorla, Basel, Heidelberg, in Mexico (UNAM) and in China, Red Purple Observatory in Nanjing, and Shanghai/Sheshan. The total number of plate scans obtained during these visits amounts to 50000 .

There is no complete inventory of all astronomical photographic records available worldwide. The wide field astronomical plate database WFPDB includes only collections wide field sky images (but there are numerous small narrow-field photographic plates as well taken at focal planes of large telescopes before the CCD era) end even that is incomplete. In the USA, the inventory of north American plates was compiled by Robbins and Osborn (2009), but the list is again far from complete. Our experience shows that there are numerous astronomical plate collections, some of them large, at places and Institutions unknown to the community before. Some of them were visited by us with test plate scanning using our transportable device, described in the next section. The plate archive of the Tuorla Observatory in Finland with 11000 negatives was digitized fully within one week.

\section{Photographic plate scanning methods}

As already mentioned, before the digitization era, the access to the data recorded in astronomical photographic plates and negatives was laborious. Recently, there are digitization efforts at several observatories, but the data in vast majority of them are still unavailable. The three basic plate scanning techniques developed and tested over last decade are as follows.

- Custom made high quality scanner, mostly with granite table. Preference: very high astrometric accuracy 0.1 microns or less, drawbacks: very expensive device, expensive operation, plate scanning cost estimation 100 Euro per plate or more. This method is used e.g. in digitization of the $\mathrm{HCO}$ plate collection.

- Commercial high quality flatbed scanner. Most used instrumentation in plate scanning. Moderately expensive investment and operation, one plate scan typically of order of 10 Euro. Usually good accuracy in one direction but worse in the direction of CCD line movement where typically waves are detectable. This method is in common use e.g. at the Sonneberg Observatory (Hippke et al., 2017) and in the German APPLAUSE project (https://www.platearchive.org/applause).

- High quality digital camera with homogeneous light table and high quality lens. Moderate expensive equipment but operation is extremely fast typically one plate just very few seconds 
and hence very inexpensive. Typical cost of digitization is just 0.5 Euro or even less. Suitable for medium sized up to small and very small plates where the method provides very good resolution and accuracy.

The digitization of the archival astronomical plates is affected by many additional and specific issues such as cracked plates and plates covered densely by marks (Figs. 1 and 2).

Some recent extended digitization projects include automated pipeline, e.g. APPLAUSE (Germany Hamburg, Bamberg, Potsdam) with 72314 plates so far, https://www.plate-archive.org/applause/, and DASCH HCO USA with 324908 scanned plates so far, affected by flooding few years ago (scanner lost), http://dasch.rc.fas.harvard.edu/lightcurve.php.

\section{In situ digitization with transportable device}

Most of the plate archives that we visited have no plate scanners and lack modern instrumentation in general. As our study included plate digitization, it was necessary to find a solution. The obvious option was a transportable digitization device based on a digital camera with a highquality lens and a stable tripod. This solution has the following advantages over other techniques: the device is easily transportable, and offers much faster scanning and higher repeatability than commercial flatbed scanners, because there are no moving scanner parts. The equipment that we used at the beginning was as follows: Camera: 21 MPx Canon EOS 5D Mark II, Lenses: Canon EF 24-70 f/2.8 L USM and Canon 70-200mm F4, a stable tripod, and a Fomei LP-310 professional photographic light table. More recently, a better custom-made light table based on highly homogeneous LED illumination was used, and also a further improved camera (39 Mpx) and lenses. The recorded images are then corrected for lens image distortions and for other effects, in order to store research-grade digital images. The achieved pixel size depends on the size of the plate, and fine pixel sizes of order of 25-30 microns or less can be typically obtained for small and medium sized plates (up to $16 \mathrm{~cm}$ x $16 \mathrm{~cm}$ or $13 \mathrm{~cm}$ x $13 \mathrm{~cm}$ ) while for larger plates larger pixel sizes will be obtained (or, alternatively, the large plate may be covered by several shots to achieve smaller pixel sizes).

The transportable scanning device described above represents cost effective and fast digitization method. It should be taken into account that numerous plate collections were found to be affected by degradation, in some cases progressing, e.g. gold decease (Fig. 3) so that the fast scanning is necessary to avoid the lost of scientific information recorded in these images.

The example of an astrometric solution for CdC plate UNAM Mexico digitized by digital camera is shown in the Fig.4 with the calculated parameters as follows. Center (RA, Dec): (178.349, -11.387), Center (RA, hms): 11h 53m 23.650s, Center (Dec, dms): -11 deg 23 arcmin 12.371 arcsec, Size: 1.79 x $2.01 \mathrm{deg}$, Radius: $1.345 \mathrm{deg}$, Pixel scale: $1.96 \mathrm{arcsec} / \mathrm{pixel}$.

\section{Conclusions}

The digitization of astronomical photographic archives is ongoing in the USA (HCO) and in Germany (Bamberg, Hamburg, Potsdam) and there are also automated pipelines to evaluate these data. 


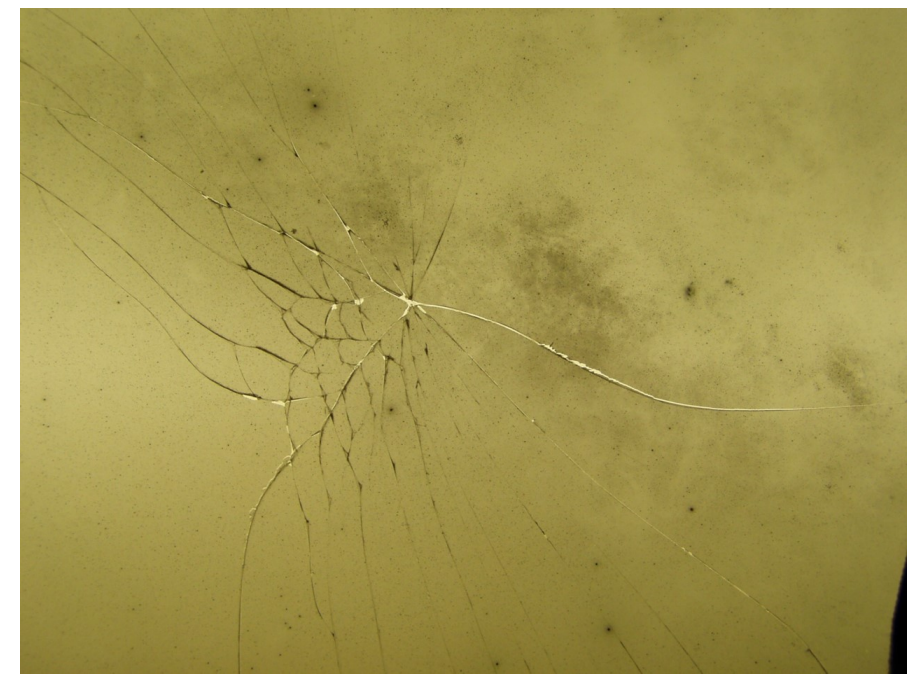

Figure 1: The cracked plate from the $\mathrm{HCO}$ archive. Such plates represent an issue during digitization process.

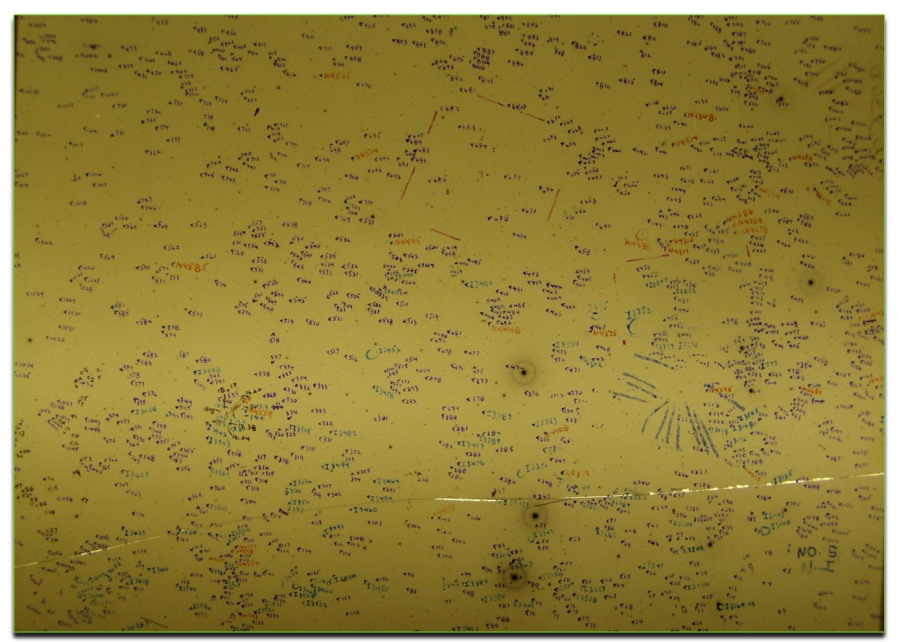

Figure 2: The heavily investigated photographic plate with dense marks. Such plates represent an issue during digitization process. Removing the marks can be, in some cases, related to lost of information.

On the other hand, the digitization efforts are still limited to few places. It is obvious, that, taking the huge number of astronomical archival plates and negatives as well as limited resource into account, we need inexpensive and fast in situ digitization technique. The alternative plate scanning method based on transportable device was developed and tested. This device was then further improved and upgraded including special software for automated image treatment and lens distorsion correction etc. The astrometric and photometric accuracy of the method was tested and compared, where possible, with other plate scanning methods. The method is fast and inexpensive still yielding scientific grade results.

Selected plates were digitized using a transportable scanning device. All the archives that we visited have plates that are scientifically valuable, and in many cases unique. The plates are however 


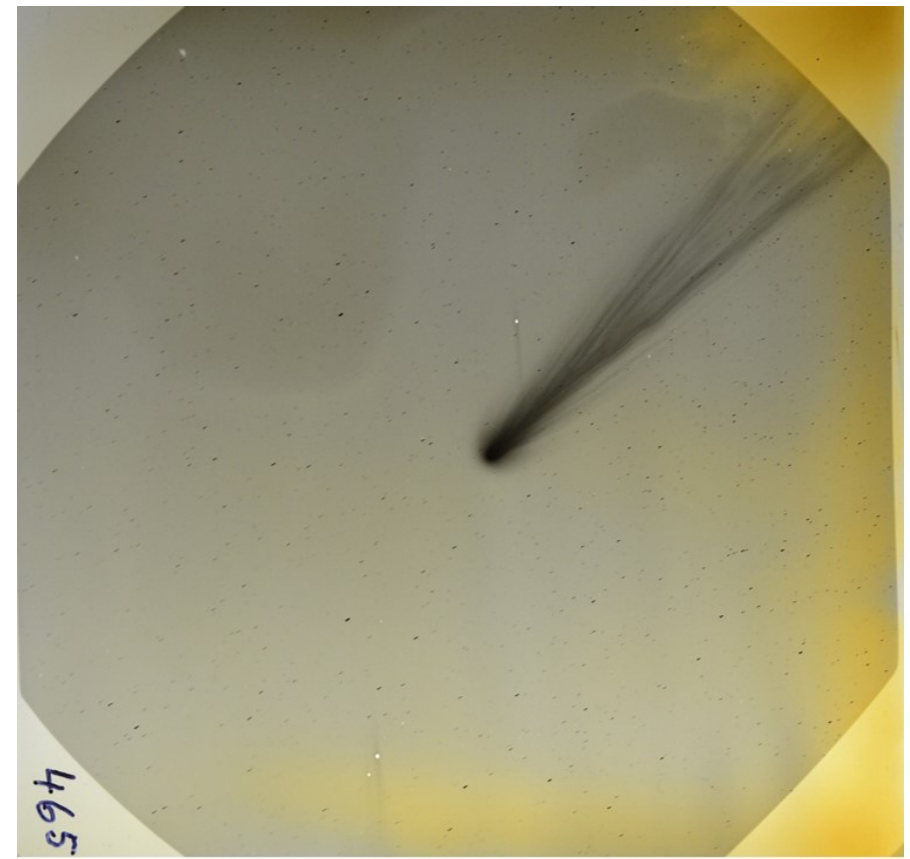

Figure 3: The unique image of the Halley comet from the Klet Observatory plate archive. The plate degradation by golden decease is clearly visible. This degradation type was found in numerous photographic plate archives.



Figure 4: The astrometric solution for the UNAM CdC plate digitized by digital camera provided by astrometry.net. See text for details. 
often hidden from the astronomical community, and the plates have not yet been catalogued.

\section{Acknowledgements}

We acknowledge GA CR grant 13-39464J. We thanks Dr. Taavi Tuvikene for his help with astrometry accuracy analysis and Dr. Corine Rossi for valuable comments for the paper improvement.

\section{References}

[1] Hudec, R. et al., Acta Polytechnica, IBWS2011 Proceedings, 1(52), 2012.

[2] Hudec, R. Astrophysics with Astronomical Plate Archives, in Exploring the Cosmic Frontier: Astrophysical Instruments for the 21st Century. ESO Astrophysics Symposia, European Southern Observatory series. Edited by Andrei P. Lobanov, J. Anton Zensus, Catherine Cesarsky and Phillip J. Diamond. Series editor: Bruno Leibundgut, ESO. ISBN 978-3-540-39755-7. Published by Springer-Verlag, Berlin and Heidelberg, Germany, 2007, p.79

[3] Hudec, R., An introduction to the world's large plate archives, Acta Historica Astronomiae, vol. 6, p. 28-40, 1999.

[4] Robbins, L.; Osborn, W., The Census of Astronomical Photographic Plates in North America, Preserving Astronomy's Photographic Legacy: Current State and the Future of North American Astronomical Plates. ASP Conference Series, Vol. 410. Edited by Wayne Osborn and Lee Robbins. San Francisco: Astronomical Society of the Pacific, 2009., p.81

[5] Hudec, R., World's Astronomical Plate Archives: Past, Present, Future in Virtual Observatory: Plate Content Digitization, Archive Mining and Image Sequence Processing, iAstro workshop, Sofia, Bulgaria, 2005 proceedings, ISBN-10 954-580-190-5, p. 197-208

[6] Hudec R. and Hudec L., Finding Hidden Treasures: Investigations in US Astronomical Plate Archives Acta Polytechnica, Vol. 53, No. 3, p.23, 2013 [4]

[7] Hippke, M., P. Kroll, F. Matthai, D. Angerhausen, T. Tuvikene, K. G. Stassun, E. Roshchina, T. Vasileva, I. Izmailov, N. N. Samus, E. N. Pastukhova, I. Bryukhanov, and M. B. Lund: Sonneberg Plate Photometry for BoyajianâĂŹs Star in Two Passbands, The Astrophysical Journal, Volume 837, id. 85 (2017). 